Bond University

Research Repository

\title{
Food for Thought
}

\section{A Randomised Controlled Trial of Emotional Freedom Techniques and Cognitive Behavioural Therapy in the Treatment of Food Cravings}

Stapleton, Peta; Bannatyne, Amy Jean; Urzi, Keri Charle; Porter, Brett; Sheldon, Terri

Published in:

Applied Psychology: Health and Well-Being

DOI:

10.1111/aphw. 12070

Link to output in Bond University research repository.

Recommended citation $(A P A)$ :

Stapleton, P., Bannatyne, A. J., Urzi, K. C., Porter, B., \& Sheldon, T. (2016). Food for Thought: A Randomised Controlled Trial of Emotional Freedom Techniques and Cognitive Behavioural Therapy in the Treatment of Food Cravings. Applied Psychology: Health and Well-Being, 8(2), 232-257. https://doi.org/10.1111/aphw.12070

\section{General rights}

Copyright and moral rights for the publications made accessible in the public portal are retained by the authors and/or other copyright owners and it is a condition of accessing publications that users recognise and abide by the legal requirements associated with these rights.

For more information, or if you believe that this document breaches copyright, please contact the Bond University research repository coordinator. 
Food for Thought: A Controlled Trial of Emotional Freedom Techniques and Cognitive Behavioural Therapy in the Treatment of Food Cravings

\begin{abstract}
Addressing the internal determinants of dysfunctional eating behaviours (e.g., food cravings) in the prevention and treatment of obesity has been increasingly recognised. This study compared Emotional Freedom Techniques (EFT) to Cognitive Behavioural Therapy (CBT) for food cravings in adults who were overweight or obese $(N=83)$ in an 8-week intervention. Outcome data was collected at baseline,, post-intervention, and at six and 12months follow-up. Overall, EFT and CBT demonstrated comparable efficacy in reducing food cravings, one's responsiveness to food in the environment (power of food), and dietary restraint, with Cohen's effect size values suggesting moderate to high practical significance for both interventions. Results also revealed both EFT and CBT are capable of producing treatment effects that are clinically meaningful, with reductions in food cravings, the power of food, and dietary restraint normalising to the scores of a non-clinical community sample. While reductions in BMI were not observed, the current study supports the suggestion psychological interventions are beneficial for food cravings and both CBT and EFT could serve as vital adjunct tools in a multidisciplinary approach to managing obesity.
\end{abstract}

Keywords: food cravings, obesity, cognitive behavior therapy, emotional freedom techniques 
Food for Thought: A Controlled Trial of Emotional Freedom Techniques and Cognitive Behavioural Therapy in the Treatment of Food Cravings

According to the World Health Organisation (WHO), obesity has become a vast global epidemic, with prevalence rates more than doubling between 1980 and 2014 (WHO, 2014). By 2030, the percentage of individuals classified as overweight or obese range globally is expected to increase to 57.8 percent, an increase of 5.8 percent over a 15 year period (Kelly, Yang, Chen, Reynolds, \& He, 2008). With no indication of this trend ceasing, and increasing evidence that the number of individuals falling within the overweight and obese categories is growing, the importance of addressing issues contributing to obesity (directly or indirectly) is clear (Aitken, Allman-Farinelli, King, \& Bauman, 2009). Although efforts to address environmental factors and behaviours contributing to obesity are underway, research has highlighted the importance of addressing the internal determinants of dysfunctional eating behaviours in the prevention and treatment of obesity (Buckroyd \& Rother, 2008).

The presence of food cravings, for instance, has been shown to serve as an important factor influencing appetite control (Blundell et al., 2005). Food cravings generally refer to a motivational state in which the individual feels an intense desire to seek and consume a specific food (Tiggemann \& Kemps, 2005). Although food cravings have been associated with hunger and at times attributed to nutritional deficits (Wansink, Cheney, \& Chan, 2003), nutritional deprivation is not a necessary condition for food cravings to occur. Other precipitating factors include negative mood states (Lafay et al., 2001), menstrual-related fluctuations (Zellner, Garriga-Trillo, Centeno, \& Wadsworth, 2004), and cognitive factors such as expectancies (Haddock \& Dill, 2000).

Food cravings also play an important role in excess energy intake and weight gain (Moreno et al., 2008; Steel, Kemps, \& Tiggemann, 2006), increases in BMI (Delahanty et al., 2002; Franken \& Muris, 2005), lack of weight loss success (Jakubowisz, Froy, Wainstein, \& 
Boaz, 2011), binge eating (Gendall, Joyce, Sullivan, \& Bulik, 1998) and early dropout from weight-loss interventions (Lim, Norman, Clifton, \& Noakes, 2009; Moreno et al., 2008). Even in non-clinical samples, food cravings have been positively correlated to body weight, highlighting the considerable role of cravings in food consumption (Franken \& Muris, 2005).

The physiological effects of being overweight or obese are well established. Obesity is a causative factor increasing an individual's risk of developing cardiovascular disease, Type II diabetes, musculoskeletal disorders, and premature mortality (See Reilly \& Kelly, 2011, for a full review). The increasing healthcare cost of obesity has also been highlighted in a recent systematic review (e.g., Withrow \& Alter, 2010), with obesity reportedly accounting for 0.7 to 2.8 percent of total healthcare expenditures, depending on the country.

Given the significant impact of obesity, on both individuals and the community, considerable resources have been devoted to developing effective weight control interventions in recent years. Effectively managing obesity, however, is widely acknowledged to be a difficult problem (Turk, Yang, Hravnak, Sereika, Swing, \& Burke, 2009). More specifically, behavioural weight loss programs (e.g., dietary restraint and physical exercise), long purported to be the solution to the obesity crisis, have demonstrated modest short-term effectiveness (Wadden, Steen, Wingate, \& Foster, 1996), but poor longterm maintenance (Turk et al., 2009). For example, meta-analytic and longitudinal studies indicate between one to two thirds of the weight lost through behavioural weight reduction programs is regained within a year, and almost all weight is typically regained three years post-intervention (Mann, Tomiyama, Westling, Lew, Samuels, \& Chatman, 2007). One possible explanation for this poor weight loss retention is the simultaneous increase in the frequency of food cravings with dieting attempts (particularly those based on principles of 'eat less and exercise more'), suggesting that food cravings might serve as an important barrier to weight loss (Jackubowisz et al., 2011; Massey \& Hill, 2012). 


\section{Psychological Interventions for Food Cravings}

Despite the issues related to long-term weight loss maintenance, meta-analytic studies of weight programs have revealed that individuals who are overweight or obese do benefit from psychological intervention to enhance weight reduction and reduce food cravings, particularly cognitive-behavioural strategies (Ismail, Winkley, \& Rabe-Hesketh, 2004; Shaw, O’Rourke, Del Mar, \& Kenardy, 2005). CBT approaches targeting obesity not only focus on addressing an individual's overeating or low level of activity, but also the mechanisms hypothesised to perpetuate weight gain or prevent successful weight loss maintenance (Cooper \& Fairburn, 2001). The failure to address underlying cognitive (i.e., distorted thought patterns) and emotional factors has been shown to be an important barrier to achieving clinically significant treatment gains (Blundell et al., 2005). In terms of efficacy, Wilson and Fairburn (2007) found that cognitive-behavioural strategies for emotionally driven or craving-induced eating were associated with significant decreases in the frequency of these eating behaviours, with up to half of patients in this study ceasing to binge altogether.

Despite the efficacy of cognitive behavioural strategies for bulimia nervosa and binge eating disorder, therapeutic approaches have been relatively ineffectual in relation to food cravings in both clinical and community samples (Kemps \& Tiggemann, 2010). A Cochrane review (Shaw et al., 2005) highlighted that CBT and behaviour therapy (BT) significantly improved the success of weight loss for overweight and obese research participants, while cognitive therapy (CT) alone was not found to be effective as a weight loss treatment. Similarly, there appears to be a paucity of research examining the use of other psychological interventions (other than CBT) in the treatment of food cravings or weight loss interventions (Shaw et al., 2005). Moreover, literature suggests that the long-term effectiveness of CBT for weight concerns is limited (e.g., Schuurmans et al., 2009). Therefore, novel and distinctive 
craving reduction strategies could serve as vital therapeutic tools in reducing the impact of obesity. However, longitudinal evaluation of these techniques in relation to weight and BMI reduction/management is required (Stapleton, Sheldon, \& Porter, 2012).

\section{A Novel Approach to Reducing Food Cravings}

One novel treatment approach that has been receiving increasing attention is Emotional Freedom Techniques (EFT). EFT combines psychological methods (e.g., cognitive and exposure techniques), with stimulation of the “meridians” using acupuncture points (acupoints; Stapleton et al., 2012). The meridians are thought of in the practice of acupuncture as pathways for the body's subtle energy system, and techniques such as EFT that utilise the meridians for psychological change typically fall within the categorisation of “energy psychology”. Rather than using acupuncture needles, EFT has clients tap, usually with two fingers, in the area of acupoints on the face and upper body (Craig, 2010).

While the mechanisms that would account for EFT’s encouraging clinical outcomes have not been definitively established, the technique appears to rapidly produce desired changes in the neurochemistry maintaining the problem being addressed. This generic effect would appear to explain EFT's demonstrated success in treating a range of conditions (Feinstein, 2012). For example, EFT has been successful in treating a range of psychological conditions including generalised and specific anxiety, phobias, depression, post-traumatic stress disorder (PTSD), chronic pain, addiction, and emotional eating and food cravings (Church, 2013; Feinstein, 2012). Treatment length has typically varied between one and ten sessions, with phobias effectively treated within one EFT session and PTSD requiring between four and ten (Church, 2009, 2011). Research has also indicated that treatment gains persist over time, including periods of one to nine months (Church, 2010; Rowe, 2005; Wells et al., 2003). 
In addition to psychological conditions, EFT has also been evaluated for its effect on psychological wellbeing, a range of physical conditions (e.g., skin disorders, fibromyalgia), and athletic performance (see Church, 2013, for a full review).

\section{EFT and Food Cravings}

A study of 216 healthcare workers revealed that cravings for chocolate, food in general, alcohol, and tobacco decreased significantly from pre- to post-treatment, following a single, full-day EFT group workshop (Church \& Brooks, 2013). In an internet-based study of EFT and weight loss, which examined a range of affective and craving symptomatology in 72 adults enrolled in a six week online program to address emotional eating, a significant decrease in depressive symptoms and body weight (12 lbs. during first 6 weeks and a further 3 lb. in the subsequent 6 months) was observed (Church \& Wilde, 2013). Despite the need for more robust methodology (e.g., randomised controlled trials [RCTs]) acknowledged by the researchers, findings of these studies provided preliminary evidence for the effectiveness of EFT in the treatment of weight loss and food cravings.

An RCT conducted by Stapleton, Sheldon, Porter, and Whitty (2011) evaluated the efficacy of a four-week EFT group program in the treatment of food cravings in overweight or obese adults against a wait-list control across several time points (baseline, post, 6 and 12 month follow-ups). More specifically, the four-week program involved: (1) psychoeducation about EFT and how it works; (2) the nature of food cravings and how they can be addressed using the EFT tapping sequence; (3) feelings and food; and (4) relapse prevention, and using EFT for stress, relaxation, and goal setting. Results of the study revealed EFT was associated with significant improvements in food cravings, the subjective power of food, and craving restraint, from baseline to immediately post-intervention when compared to a waitlist control. The waitlist condition received the EFT treatment immediately after their data was obtained for comparison purposes and the total sample were then analysed for the EFT treatment 
effect. This improvement in food cravings and the subjective power of food was maintained at the six-month follow-up period, with a delayed positive effect observed for food restraint. Whilst no improvements in BMI or body weight were observed immediately following the EFT intervention or even at six months, a significant reduction in BMI, body weight, and psychological distress was observed at the 12-month follow-up (Stapleton et al., 2012). Improvements in food cravings, subjective power of food, and restraint were also maintained at 12-month follow-up. However, despite the promising nature of the findings, the comparison condition was a waitlist control rather than an established empirically supported treatment.

\section{The Current Study}

Given the emerging body of empirical literature surrounding EFT, the current study sought to demonstrate that the experimental treatment (EFT) was not unacceptably less efficacious than a standard treatment (CBT) for reducing food cracings (non-inferiority trial design). It is anticipated EFT as a clinical intervention may have entail certain advantages over current treatment such as skill practicality, reduced cost, or greater ease of administration outside of structured therapy. It was anticipated that after evidence of EFT being a non-inferior technique has been established that further research may explore whether EFT is a potentially superior therapeutic option.

As such, the main aim of the current study was to compare group based delivery of EFT and CBT in the treatment of food cravings with a sample of overweight and obese adults, while examining the effectiveness of both interventions over several time points (pre, post, 6 and 12 month follow-up). An additional aim of the current study was also to determine whether any reductions observed were clinically meaningful by comparing the results of the active interventions in a non-clinical community sample. Based on previous research, it was hypothesised that: 
1. Participants in both intervention conditions would exhibit a significant reduction in food cravings, restraint, and the subjective power of food, immediately post-intervention and any treatment gains would be maintained at the six and 12-month follow-ups.

2. For both intervention conditions, a delayed effect would be observed for BMI. That is, no significant reduction was expected from baseline to post-intervention or the sixmonth follow-up; however, a significant decrease was expected from baseline to the 12month follow-up for both groups.

3. EFT would demonstrate equal effectiveness in treating food cravings when compared to CBT. That is, there would be no statistically significant difference between the interventions at any time point.

\section{Method}

Prior to the study being undertaken, appropriate ethical approval was granted.

\section{Recruitment}

A purposive sample was recruited via community announcements in print advertisement, radio, and television. To be eligible for the current study, participants were required to be over 18 years of age (for consent issues); not suffering from any severe psychological issues (i.e., psychotic symptoms), as indicated via administration of the Symptom Assessment-45 (SA-45; Strategic Advantages, 2000); not receiving current treatment (psychological or medical) for his/her food cravings; have a BMI greater than 25; experiencing food cravings of a certain severity (described below); and agreed to participate in follow-up measurements. Both males and females were eligible for the intervention. Current and immediate past sufferers of bulimia nervosa and anorexia nervosa (whether clinically diagnosed or not) were excluded based on active diagnoses revealed via administration of the SA-45. Women who were pregnant and known sufferers of diabetes (Type I and II) and hypoglycemia were also excluded due to any physiological effects that 
may have suppressed feelings, cravings, and sensitivity. Unsuitable or superfluous participants were advised in letterform they did not meet selection criteria or that limited funding prevented selection. A list of suitable practitioners within the community was also provided to enable these ineligible participants to seek further treatment for their food cravings should they desire.

A total of 207 potential participants responded to the initial community announcements, with 177 screened via a telephone interview for eligibility. Applicants were screened on a first come, first served basis, with the location of treatment and funding limitations restricting the number of trial positions. Of the 177 screened, 94 were excluded for not meeting specified eligibility criteria $(n=57)$ and 37 declined to participate. The remaining were offered a place in the trial 83 were randomised via a computerized randomnumber generator system, to an eight-week EFT intervention $(n=51)$ or eight-week CBT treatment $(n=34)$. A statistician unconnected to the study and blind to its aims completed the computer randomisation (See Figure 1 for consort diagram). A community sample of 92 individuals was also recruited to serve as a normal weight non-clinical comparison to determine whether treatment gains were clinically meaningful. The community sample did not receive an active intervention and was recruited through advertisements on the social media webpages of local community groups.

\section{INSERT FIGURE 1 HERE}

Potential intervention participants were sent a pre-intervention questionnaire package consisting of demographic questions and various psychometric instruments (described below). The heights (stadiometer) and weights (electronic scale) of intervention participants were measured in person by the principal investigator at baseline and immediately post intervention; however, participants were required to self-report weight at the six-month follow-up. All participants gave informed consent. The university ethics committee approved 
the research and the study was registered under the Australia New Zealand Clinical Trials Registry.

\section{Participants}

A power analysis using $G^{*}$ Power (Faul et al., 2007) indicated a minimum of 34 participants per intervention group to detect medium effect size with $87 \%$ power $(\alpha=.05)$. The current sample satisfied this. Demographic information for all groups can be seen in Table 1. Baseline data revealed chocolate was the most commonly craved food by the intervention sample (53\%), followed by sweet carbohydrates (e.g., cakes, biscuits, soft drink) (15.7\%), carbohydrates that are neither sweet nor salty (e.g., bread) (15.7\%), salty foods (e.g., chips, salted nuts) (14.5\%), and caffeinated items (1.2\%). The majority of intervention participants reported they experienced cravings on a daily basis (83.1\%), with 67.5 percent of the sample indicating they would consume their stated craving on a daily basis. Approximately 13.3 percent reported they would consume their craved food two to six times per week.

\section{INSERT TABLE 1 HERE}

\section{Measures}

Demographic Questions. For the purpose of describing the sample, participants were asked to provide information regarding age, gender, marital status, number of people in their household, country of birth, income, and highest level of completed education.

Anthropometric Measures. Height and weight measurements were obtained to calculate BMI, which was defined as weight in kilograms divided by height in meters squared $\left(\mathrm{kg} / \mathrm{m}^{2}\right)$. BMI categories utilised for the present study included: underweight $(<18.5)$, healthy weight (18.5 to 24.9 ), overweight (25.0 to 29.9 ), or obese ( $\geq 30.0$ ).

Food Craving Inventory (FCI; White et al., 2002). The FCI is a 28-item self-report scale designed to measure cravings for 28 specific foods, over the past month, on a five-point 
Likert scale $(0=$ never and $4=$ always/almost every day $)$. Craving was defined as "an intense desire for a specific food (or food type) that is difficult to resist”, which is consistent with definitions used previous research. The FCI contained four subscales including High Fats (e.g., bacon and fried fish), Sweets (e.g., brownies and ice-cream), Carbohydrates/Starches (e.g., sandwich bread, baked potato, and pasta) and Fast Food Fats (e.g., pizza and french fries). For the purpose of the current study, the FCI Total Score was utilised, with higher scores indicative of greater food craving symptomatology.

The FCI has been found to be a valid and psychometrically sound instrument for general and specific food cravings, with good internal consistencies ranging from .70 (fast food) to .91 (high fats) for the subscales and .86 to .87 for the total scale (White et al., 2002; White \& Grilo, 2005). The FCI has been shown to have adequate temporal stability and discriminant validity, and good sensitivity to changes across treatment (White et al., 2002).

Power of Food Scale (PFS; Lowe et al., 2009). The PFS is a 21-item self-report measure, designed to assess individual differences in the anticipation (but not consumption) of highly palatable foods in the environment, for reasons other than physiological hunger (Witt \& Lowe, 2014; If I see or smell a food I like, I get a powerful urge to have some). Items were scored on a five-point Likert scale ranging from one (do not agree at all) to five (strongly agree). This measure was selected because developing control over one's eating environment has been shown to be an influential factor in long-term weight loss and maintenance (Stroebe, Papies, \& Aarts, 2008). It should be noted, however, that the items did not explicitly refer to volume of food consumed or loss of control. A total score was utilised for analysis, with high scores indicative of greater responsiveness to an individual's food environment. The PFS has been shown to be a valid and psychometrically sound instrument, with studies demonstrating adequate internal consistency, temporal stability, and convergent validity (Cappelleri et al., 2009; Lowe et al., 2009). 
Revised Restraint Eating Scale (RRS; Herman \& Polivy, 1980). The RRS is a 10item self-report measure that assesses dietary restraint as an enduring eating pattern, typically encompassing restrictive behaviours and periods of excessive eating (Heatherton, Herman, Policy, King, \& McGree, 1988). The RRS consists of two subscales: weight fluctuation (4 items assessing history of weight fluctuations) and concern with dieting (6 items assessing attitudes toward dieting). All items are scored on a four- to five-point Likert scale, with a maximum total score of 35. In the current study, a total score was utilised for analyses. Higher scores were indicative of chronic dieting, whereby the individual's lifestyle was characterised by constant cycling on and off a diet/s without substantial weight loss. Cut off scores of 16 or greater were also used to identify restrained eaters or dieters in females, and scores of 12 or greater utilised to identify restrained eaters in males. This measure was included as high dietary restraint has been shown to play a role in the development of eating disorders, obesity, and loss of control over food intake (Fedoroff, Polivy, \& Herman, 1997; Overduin \& Jansen, 1996). Food cravings (via external regulation and the presence of food cues) are believed to trigger these behaviours (Stapleton et al., 2011).

The RRS is a valid and psychometrically sound instrument, with studies revealing good internal consistencies (Davis \& Katzman, 1998; Klem, Klesges, Bene, \& Mellon, 1990; Laessle, Tuschk, Kotthaus, \& Pirke, 1989), adequate temporal stability, and external validity via convergence with the EAT-26 and discrimination with the Motivation for Eating Scale (Mak \& Lai, 2012).

\section{Treatment Conditions}

Treatment protocols and fidelity plans for EFT and CBT were formed prior to the trial commencing. The principal investigator briefed practitioners and each practitioner reviewed the relevant treatment protocols. Practitioners were not blind to their intervention delivery, but were blinded to the comparison aims of the study. Both interventions were offered in 
groups of 10 to 15 participants between 2011 and 2014, and were facilitated by trained practitioners (two for each group) at a local psychology clinic. The interventions consisted of eight, weekly sessions (two hours duration each), delivered during the hours of 6.00pm to 8.00pm. This was designed to accommodate employment and family commitments characteristic of the age demographic to prevent attrition over the duration of the treatments. A similar session structure was utilised for both interventions, with home activities included in both approaches. Although the weekly session topic for both groups was identical, it is important to note that the two groups differed in the sense that content was presented in relation to the intervention technique. For example, stress-related content presented in Week 5 was addressed by tapping on stress-related cognitions for the EFT group, and by implementing a cognitive restructuring technique for stress-related cognitive distortions for the CBT group.

Clinical EFT Treatment. For the EFT group, the practitioners were certified and experienced in EFT. Treatment was based on standardised protocols (See Craig \& Fowlie, 1995). Acupressure points on the eyebrow, side of eye, under eye, under nose, chin, collarbone, under arm, and the top of the head were used. Treatment sessions involved direct exposure to craved foods. EFT techniques were utilised by participants focusing on a specific craving and associated emotion, rating the intensity of the craving using subjective units of distress (SUDs), initiating a set-up statement, and then completing the tapping process on the specific acupoints. The setup phrase focused on the individual's difficulties and was expressed aloud in the group, adding a voiced statement of self-acceptance.

For example, if chocolate craving is experienced, a setup phrase could be "Even though I'm having a strong craving for chocolate, I deeply and completely accept myself'. This set up phrase is typically stated and repeated three times while tapping the "karate chop" point below the little finger on either hand (Craig, 2010). The tapping sequence is then 
initiated with cognitive, affective, and sensory information elicited from the individual voiced aloud while each acupoint is tapped approximately seven times each. After each tapping cycle, participants rate their SUDs and the process is repeated until the discomfort score is zero. New setup statements may be performed if pertinent information is elicited in initial rounds of tapping (e.g., “Even though I feel deprived when I don't have chocolate, I choose to be fit and healthy”). Full instructions and safeguards are described in Flint, Lammers and Mitnick (2005). Participants were encouraged to self-administer EFT outside of treatment sessions in response to cravings.

CBT Treatment. For the CBT group, the practitioners were trained psychologists, and treatment was based on standardised protocols (See Fairburn, Marcus, \& Wilson, 1993) and guided by the National Medical Health and Research Council (2013) recent clinical practice guidelines. The CBT intervention aimed to modify eating, thinking, and activity levels by teaching group members cognitive (e.g., restructuring of urge-related thoughts and/or core beliefs, mindfulness techniques) and behavioural strategies (e.g., increasing awareness through food diaries, relaxation skills, distress tolerance, problem solving, and self-esteem exercises). Similar to the EFT intervention, the CBT intervention also involved direct exposure to craved foods to elicit associated emotions and cognitions, in addition to homework tasks (e.g., thought records).

The major difference between the two treatments is the addition of the somatic tapping process in the EFT treatment. EFT's use of acupressure points is the primary distinguishing factor from treatments such as CBT. Pressure on acupoints has been found to be as efficacious as acupuncture (Cherkin et al., 2009).

\section{Design and Analysis}

A controlled non-inferiority trial was performed comparing group-delivered CBT to group-delivered EFT. To address the research question, quantitative statistical methods were 
used. Descriptive statistics were used to detail study variables. A series of chi-square analyses were performed to determine whether the groups differed based on demographic variables. A mixed (between-within subjects) 2 x 4 multivariate analysis of variance (MANOVA) was conducted to determine whether the two interventions (EFT vs CBT) had an effect on the dependent variables (e.g., FCI, POF, RRS, and BMI) combined, over time (pre-intervention vs immediately post-intervention vs 6-month follow-up vs 12 month follow-up). To establish the clinical impact of the treatments, an additional $3 \times 4$ between-subjects MANOVA was conducted to determine the differences between the two intervention groups and a nonclinical community sample. The main objective of this secondary analysis was to determine whether any reductions in scores over time were comparable to a non-clinical sample. For the purpose of the current study, only participants who completed all measurement time points were included in the final analysis.

\section{Results}

The data were analysed using SPSS (Version 23). An alpha level of .05 was utilised to determine the statistical significance of all results.

There was an average attendance rate of 6.54 sessions for the total sample. Based on efficacy approaches when analysing clinical trials, an "as treated” approach was taken by incorporating only participants who attended six or more sessions (i.e., at least $80 \%$ of the treatment; Armijo-Olivo, Warren, \& Magee, 2015).

\section{Sample Characteristics}

In terms of treatment assignment and follow-up, 47 participants completed the EFT intervention, while 34 were completed the CBT intervention. At the 6- and 12-month follow-up periods, 37 (78\%) and 32 (68\%) EFT participants completed the follow-up questionnaires, respectively. For CBT, 29 (85\%) and 24 (71\%) completed the follow-up 
questionnaires, respectively. As such, the final analysis included 32 EFT participants and 24 CBT participants.

Results of chi-square analyses revealed no significant differences between groups in relation to gender $\chi^{2}(1)=.24, p=.622$; relationship status $\chi^{2}(5)=6.37, p=.272$; age $\chi^{2}(8)=$ $6.80, p=.250$; education level $\chi^{2}(6)=14.13, p=.118$; income $\chi^{2}(10)=7.81, p=.648$; or craving type $\chi^{2}(4)=3.56, p=.468$. As can be seen in Table 2, at pre-intervention both intervention groups had mean BMI scores in the “obese” range, high levels of dietary restraint, and a high level of responsiveness to food in the environment (i.e., power of food). In summary, the two treatment groups did not differ significantly at baseline. Furthermore, results of several independent t-tests revealed there were no significant differences in baseline FCI, POF and RRS scores, or BMI, between those who did (completers) and did not complete (drop outs) the active interventions.

\section{INSERT TABLE 2 HERE}

\section{Non-Inferiority Analyses: CBT vs EFT}

With the use of Wilk's criterion, a significant multivariate main effect was found for Time $F(12,43)=9.35, p=<.001$, partial $\eta^{2}=.72$, but not for Group $F(4,51)=.42, p=.797$, partial $\eta^{2}=.03$, or the interaction between Time and Group $F(12,43)=1.24, p=.291$, partial

$\eta^{2}=.26$. As such, further analyses focused primarily on the main effect of Time only. It should be noted that testing for differences across time does not consider the impact of the intervention allocated as the groups are collapsed. Furthermore, as Mauchly's Test of Sphericity for each of the dependent variables indicated the assumption of sphericity had been violated, Huynh Feldt was utilised at the univariate level. Refer to Table 3 for a summary of within-group effects for each dependent variable.

\section{INSERT TABLE 3 HERE}

Food cravings. Univariate analyses revealed a significant difference in food craving 
scores across the four different time points $F(2.25,121.53)=19.38, p=<.001$, partial $\eta^{2}=$ .26. Pairwise comparisons with Sidak adjustment revealed food craving scores decreased significantly across both groups from pre-intervention to post-intervention $(p=<.001, d=$ .89), with this reduction maintained at the 6-month $(p=<.001, d=.87)$ and 12-month $(p=<$ $.001, d=.74)$ follow-up points.

Power of food., Univariate analyses revealed a significant difference in power of food scores across the four different time points $F(2.13,115.04)=26.77, p=<.001$, partial $\eta^{2}=$.33. Pairwise comparisons with Sidak adjustment revealed power of food scores decreased significantly across both groups from pre-intervention to post-intervention $(p=<$ $.001, d=.83)$, with this reduction maintained at the 6-month $(p=<.001, d=.92)$ and 12month $(p=<.001, d=.98)$ follow-up points.

Restraint. Univariate analyses revealed a significant difference in restraint scores across the four different time points $F(1.95,105.22)=13.34, p=<.001$, partial $\eta^{2}=.20$. Pairwise comparisons with Sidak adjustment revealed restraint scores decreased significantly across both groups from pre-intervention to post-intervention $(p=<.001, d=.48)$, with this reduction maintained at the 6-month $(p=<.001, d=.57)$ and 12-month $(p=<.001, d=.85)$ follow-up points. Of note, there was a significant reduction in restraint scores from the 6- to 12-month follow-up ( $p=.005, d=.005)$.

BMI. Univariate analyses revealed no significant difference in BMI scores across the four different time points $F(2.25,57.13)=.14, p=.722$, partial $\eta^{2}=.01$.

\section{Clinical Efficacy Analyses: CBT vs EFT vs Non-Clinical Community Sample}

With the use of Wilk's criterion, a significant multivariate main effect was found for Group $F(32,260)=9.74, p=<.001$, partial $\eta^{2}=.55$, indicating there was a statistically significant difference between groups on the dependent variables combined. As such univariate analyses for each dependent variable were explored with Tukey's post-hoc 
analyses. See to Table 4 for a summary of between-subjects (CBT vs EFT vs Community) univariate analyses for each dependent variable.

\section{INSERT TABLE 4 HERE}

Food cravings. At pre-intervention, significant differences in food craving scores were observed between groups, with post-hoc Tukey's analyses indicating the EFT and CBT groups had significantly higher food cravings scores than the community sample $(p=.001$ and $p=<.001$, respectively). At post-intervention, no significant differences were found between groups, indicating both intervention groups had comparable food craving scores to the non-clinical community sample. This was maintained at the 6- and 12- month follow-ups.

Power of Food. At pre-intervention, significant differences in power of food scores were observed between groups, with post-hoc Tukey's analyses revealing the EFT and CBT group had significantly higher power of food scores than the community sample $(p=<.001$ and $p=<.001$, respectively). Significant differences between groups were also observed at post-intervention; however, only the CBT group obtained significantly higher scores than the community sample $(p=.009)$. At this time point, the EFT group appeared to have comparable power of food scores to the community sample, yet there was no significant difference in scores between the intervention groups. At the 6- and 12- month follow-ups, no significant differences between groups were observed, indicating the EFT and CBT groups had comparable power of food scores to the community sample at these time points.

Restraint. At pre-intervention, significant differences in restraint scores were observed between groups, with post-hoc Tukey's analyses revealing the EFT and CBT group had significantly higher restraint scores than the community sample $(p=<.001$ and $p=<$ .001, respectively). At post-intervention, significant differences were also found between groups. Post-hoc Tukey's analyses revealed both the EFT and CBT groups obtained significantly higher restraint scores than the community sample $(p=<.001$ and $p=.003$, 
respectively). Similar results were also observed at the 6-month follow up, with both the EFT and CBT groups obtaining significantly higher restraint scores than the community sample ( $p$ $=.007$ and $p=.003$, respectively). However, at the 12-month follow-up no significant differences were observed between groups, indicating the restraint scores of both intervention groups had reduced to a level comparable to the scores of the community sample.

BMI. At pre-intervention, significant differences in BMI scores were observed between groups, with post-hoc Tukey's analyses revealing the EFT and CBT group had significantly greater BMI scores than the community sample $(p=<.001$ and $p=<.001$, respectively). At post-intervention, significant differences were also found between groups, with the EFT and CBT groups obtained significantly higher BMI scores than the community sample ( $p=<.001$ and $p=<.001$, respectively). Similar results were also observed at the 6month $(p=<.001$ and $p=<.001$, respectively) and 12-month $(p=<.001$ and $p=<.001$, respectively) follow up periods, indicating the BMI of both intervention groups did not reduce to a level comparable to the non-clinical community sample at any time point during the study.

\section{Discussion}

The present study contributes to the growing body of literature highlighting the need to scientifically explore the effectiveness of novel therapeutic approaches in the treatment of food cravings, particularly in overweight and obese populations. The present study examined the effect of EFT (a novel treatment approach) and CBT (an evidence-based treatment approach with variety of conditions) in a controlled non-inferiority trial design. An additional aim of the current study was also to explore the clinical impact of these treatments when compared to a non-clinical community sample.

\section{Comparison of Efficacy}

Overall, results of the current study revealed both the EFT and CBT programs were 
equally effective in reducing food cravings, the perceived power of food, and dietary restraint, with these treatment improvements maintained six and 12 months following the interventions, demonstrating the robustness of treatment gains. No significant differences between groups were observed on any measure at any time point during the study, providing support for our first hypothesis. In addition to comparative efficacy, results of the study are also supportive of our third hypothesis, that any treatment gains achieved would be maintained at the 6 and 12 month follow ups. As such, results of the current study are supportive of the retention of treatment effects detailed for CBT (Butler, Chapman, Forman, \& Beck, 2006) and the durability of treatment outcomes observed in other EFT trials (Feinstein, 2008; Rowe, 2005; Wells et al., 2003). However, to robustly determine the enduring nature of these interventions, longer follow-up periods are required (e.g., 5 years) with much larger sample sizes.

Despite the promising treatment impact for the psychological outcome measures, no significant changes in BMI were observed for either intervention group across time. While it was not anticipated that significant reductions in BMI would be seen until the 12 month follow up, no such decrease was revealed. As such, the results were inconsistent with our second hypothesis. Although a delayed BMI reduction was observed in Stapleton et al. (2011) at 12-months following a 4-week EFT intervention, the BMI results of the current study are not surprising given the absence of dietary or exercise instruction in the interventions. Previous research comparing CBT and CBT plus dietary and exercise instruction in 70 participants has revealed a combination of CBT and dietary/exercise guidance results in significantly greater weight reductions at 3-months post-intervention, compared to CBT alone (Painot et al., 2001). As such, it is vital future research explores the potential impact of EFT and CBT within the context of multicomponent interventions. Overall, we believe results of the current study suggest adjunctive psychological techniques 
would be beneficial in weight management interventions.

\section{Clinical Impact}

An additional aim of the current study was also to determine whether the reductions in food cravings, power of food, and dietary restraint were comparable to the scores of a nonclinical community sample within a normal BMI range. In this respect, we were interested in establishing the clinical significance of the interventions to ensure reductions observed were clinically meaningful. For total food cravings, the scores of the intervention groups were comparable to the non-clinical community sample by post-intervention, with this maintained at the 6- and 12-month follow-ups. Of interest, the scores of the EFT group were actually lower than the community sample at post-intervention and at the 6-month follow-up; however, this difference was not statistically significant. In terms of perceived power of food, the scores of the EFT group were within a non-clinical range by post-intervention; however, scores of the CBT group only became comparable to the community sample at the 6-month follow. Both intervention groups also had power of food scores comparable to the community sample at the 12-month follow-up.

For dietary restraint, the observed reductions for both interventions only became comparable to the non-clinical community sample at the 12-month follow-up. It is also important to highlight the mean score of the community sample fell within a range indicative of dietary restraint. While some studies have indicated community samples exhibit disordered eating behaviours, possibly due to socially and culturally determined perceptions of appropriate food intake and dietary ideals (Wardle, Steptoe, Oliver \& Lipsey, 2000), the absence of elevation on other variables potentially suggests the restraint scale may overpathologise eating related behaviours. Further research is needed to clarify this observation and future studies may benefit for including alternate measures of dietary restraint. 


\section{Limitations and Future Directions}

Despite the promising nature of the findings, certain limitations are noted. Firstly, the majority of participants in both groups were female and from the same community, therefore generalisability of results to males and wider community populations is limited. Similarly, the sample size was relatively small, thus reducing statistical power. Despite diligent followup procedures there was attrition during follow-up. It is unclear whether these non-responders did or did not benefit from therapeutic intervention, which may have altered the outcome of the results; however, attrition was consistent across both interventions. Future research should endeavor to rectify these sampling limitations by evaluating EFT and CBT in a larger, gender-balanced sample, ideally from multiple locations. The current study could also have been improved by incorporating an active control condition to enhance our understanding of the difference between EFT and CBT approaches, and how EFT could potentially contribute to therapeutic outcomes not covered within CBT-based treatment.

While social validity scales were incorporated into the treatment protocols, it is possible social desirability, placebo effect, therapist effects, perceived pressure to report improvements, or group dynamics influenced results. Given the current study delivered both interventions via a group format, it would be of benefit to determine if similar results are obtained via individual intervention delivery. It would also be crucial to assess or measure physical activity in future studies, as this may have been a confounding variable in the current study.

\section{Concluding Remarks}

This is the first study to examine and compare the effectiveness of EFT and CBT in reducing food cravings in a group of obese adults. Overall, findings indicated EFT and CBT were equally effective in reducing food cravings, one's responsiveness to food in the 
environment, and dietary restraint, with Cohen's effect size values suggesting moderate to high practical significance in both interventions. In addition to comparable efficacy, our results demonstrated both interventions are capable of producing treatment effects that are clinically meaningful, particularly for psychological outcomes. While reductions in BMI were not observed, the current study supports the suggestion psychological interventions are beneficial for food cravings and both CBT and EFT could serve as vital adjunct tools in the management of obesity. Further replication and longitudinal follow-up studies are required to confirm and validate the efficacy and robustness of EFT in the treatment of food cravings, and examine the mechanisms that are likely to underlie the effectiveness of EFT and how these differ to those for CBT. Ultimately it hoped that consumers in the future will have a range of management tools to select from in the worldwide battle against obesity. 


\section{References}

Aitken, R. J., Allman-Farinelli, M. A., King, L. A. \& Bauman, A. E. (2009). Current and future costs of cancer, heart disease and stroke attributable to obesity in Australia - a comparison of two birth cohorts. Asia Pacific Journal of Clinical Nutrition, 18, 63-70.

Armijo-Olivo, S., Warren, S., \& Magee, D. (2009). Intention to treat analysis, compliance, drop-outs and how to deal with missing data in clinical research: a review. Physical Therapy Reviews, 14, 36-49. doi: 10.1179/174328809X405928

Blundell, J., Stubbs, R., Golding, C., Croden, F., Alam, R., Whybrow, S., . . Lawton, C. (2005). Resistance and susceptibility to weight gain: Individual variability in response to a high-fat diet. Physiology \& Behavior, 86, 614-622.

Buckroyd, J., \& Rother, S. (2008). Psychological responses to eating disorders and obesity: Recent and innovative work. London, England: John Wiley \& Sons.

Butler, A. C., Chapman, J. E., Forman, E. M., \& Beck, A. T. (2006). The empirical status of cognitive behavioural therapy: A review of meta analyses. Clinical Psychology Review, 26, 17-31. doi:10.1016/j.cpr.2005.07.003

Cappelleri, J. C., Bushmakin, A. G., Gerber, R. A., Leidy, N. K., Sexton, C. C., Karlsson, J., \& Lowe, M. R. (2009). Evaluating the Power of Food Scale in obese subjects and a general sample of individuals: Development and measurement properties. International Journal of Obesity, 33, 913-922.

Cherkin, D. C., Sherman, K. J., Avins, A. L., Erro, J. H., Ichikawa, L., Barlow, W. E., Delaney, K., Hawkes, R., Hamilton, L., Pressman, A., Khalsa, P. S., \& Deyo, R. A. (2009). A randomized trial comparing acupuncture, simulated acupuncture, and usual care for chronic low back pain. Archives of Internal Medicine, 11:169(9), 858-866. doi: 10.1001/archinternmed.2009.65

Church, D. (2013). Clinical EFT as an evidence-based practice for the treatment of 
psychological and physiological conditions. Scientific Research, 4, 645-654.

Church, D. (2010). The treatment of combat trauma in veterans using EFT (Emotional Freedom Techniques): A pilot protocol. Traumatology, 15(1), 45-55.

Church, D., \& Brooks, A. J. (2010). The effect of a brief EFT (Emotional Freedom Techniques) self-intervention on anxiety, depression, pain and cravings in healthcare workers. Integrative Medicine: A Clinician’s Journal, 9(5), 40-44.

Church, D., Geronilla, L., \& Dinter, I. (2009). Psychological symptom change in veterans after six sessions of Emotional Freedom Techniques (EFT): An observational study. [Electronic journal article]. International Journal of Healing and Caring, 9(1).

Church, D., Piña, O., Reategui, C., \& Brooks, A. (2011). Single session reduction of the intensity of traumatic memories in abused adolescents after EFT: A randomized controlled pilot study. Traumatology. Advance online publication. doi:10.1177/1534765611426788

Church, D., \& Wilde, N. (2013). Emotional eating and weight loss following Skinny Genes: A six week online program. Proceedings of the Annual Conference of the Association for Comprehensive Energy Psychology, May 2013.

Cooper Z., \& Fairburn C. G. (2001). A new cognitive behavioural approach to the treatment of obesity. Behaviour Research and Therapy, 39, 499-511.

Craig, G. (2010). The EFT manual. Santa Rosa, CA: Energy Psychology Press.

Craig, G., \& Fowlie, A. (1995). Emotional freedom techniques: The manual. Sea Ranch, CA: Author.

Davis, C., \& Katzman, M. A. Chinese men and women in the United States and Hoong Kong: Body and self-esteem raings as a prelude to dieting and exercise. International Journal of Eating Disorders, 23, 99-102

Delahanty, L. M., Meigs, J. B., Hayden, D., Williamson, D. A., \& Nathan, D. M. (2002). 
Psychological and behavioural correlates of baseline BMI in the Diabetes Prevention Program (DPP). Diabetes Care, 25, 192-198. doi:10.2337/diacare.25.11.1992

Gendall, K. A., Joyce, P. R., Sullivan, P. F., \& Bulik, C. M. (1998). Food cravers: Characteristics of those who binge. International Journal of Eating Disorders, 23, $353-360$.

Fairburn, C. G., Marcus, M.D., \& Wilson, G. T. (1993). Cognitive-behavioral therapy for binge eating and bulimia nervosa: A comprehensive treatment manual. In C. G. Fairburn \& G. T. Wilson (Eds.), Binge eating: Nature, assessment and treatment (pp. 361-404). New York: Guilford Press.

Faul, F., Erdfelder, E., Lang, A.-G., \& Buchner, A. (2007). G*Power 3: A flexible statistical power analysis program for the social, behavioral, and biomedical sciences. Behavior Research Methods, 39, 175-191.

Fedoroff, I. C., Polivy, J., \& Herman, C. P. (1997). The effect of pre-exposure to food cues on the eating behavior of restrained and unrestrained eaters. Appetite, 28, 33-47.

Feinstein, D. (2008). Energy psychology: A review of the preliminary evidence. Psychotherapy: Theory, Research, Practice, and Training, 45, 199-213. Retrieved from http://www.innersource.net/ep/epresearch.html

Feinstein, D. (2010). The case for energy psychology. Psychotherapy Networker. Retrieved from www.psychotherapynetworker.org/magazine/currentissue/1155-the-case-forenergy-psychology

Feinstein, D. (2012). Acupoint stimulation in treating psychological disorders: Evidence of efficacy. Review of General Psychology, 4, 59-80.

Franken, I. H. A., \& Muris, P. (2005). Individual differences in reward sensitivity are related to food craving and relative body weight in healthy women. Appetite, 45, 198-201.

Greenberg, L. S. (2010). Emotion-focused therapy: A clinical synthesis. Focus, 8, 32-42. doi: 


\subsection{6/foc.8.1.foc32}

Haddock, C. K., \& Dill, P. L. (2000). The effects of food on mood and behavior: implications for the addictions model of obesity and eating disorders. Drugs \& Society, 15(1-2), 1747. doi: 10.1300/J023v15n01_02

Heatherton, T. F., Herman, C. P., Polivy, J., King, G. A., \& McGree, S. T. (1988). The (mis) measurement of restraint: An analysis of conceptual and psychometric issues. Journal of Abnormal Psychology, 97, 19-28.

Herman, C. P., \& Polivy, J. (1980). Restrained eating. In A. J. Standard (Ed.), Obesity (pp. 208-225). Philadelphia, PA: W. B. Saunders.

Hill, A. J., \& Heaton-Brown, L. (1994). The experience of food craving: A prospective investigation in healthy women. Journal of Psychosomatic Research, 38, 801-814.

Ismail, K., Winkley, K., \& Rabe-Hesketh, S. (2004). Systematic review and meta-analysis of randomised controlled trials of psychological interventions to improve glycaemic control in patients with type 2 diabetes. The Lancet, 363, 1589-1597. doi:

10.1016/S0140-6736(04)16202-8

Jakubowicz, D., Froy, O., Wainstein, J., \& Boaz, M. (2011). Meal timing and composition influence ghrelin levels, appetite scores and weight loss maintenance in overweight and obese adults. Steroids, 77, 323-331.

Kelly, T., Yang, W., Chen, C.S., Reynolds, K., \& He, J. (2008). Global burden of obesity in 2005 and projections to 2030. International Journal of Obesity, 32, 1431-1437. doi: 10.1038/ijo.2008.102

Kemps, E., \& Tiggemann, M. (2010). A cognitive experimental approach to understanding and reducing food cravings. Current Directions in Psychological Science, 19, 86-90. doi:10.1177/0963721410364494

Klem, M. L., Klesges, R. C., Bene, C. R., \& Mellon, M. W. (1990). A psychometric study of 
restraint: The impact of race, gender, weight, and marital status. Addictive Behaviours, $15,147-152$.

Laessle, R. G., Tuschl, R. J., Kotthaus, B. C., \& Pirke, K. M. (1989). A comparison of the validity of three scales for the assessment of dietary restraint. Journal of Abnormal Psychology, 98, 504-507.

Lafay, L., Thomas, F., Mennen, L., Charles, M. A., Eschwege, E., Borys, J.- M., \& Basdevant, A. (2001). Gender differences in the relation between food cravings and mood in an adult community: Results from the Fleurbaix Laventie Ville Sante Study. International Journal of Eating Disorders, 29, 195-204.

Lee, C., Colagiuri, R., Magliano, D., Cameron, A., Shaw, J., Zimmet, P., \& Colagiuri, S. (2013). The cost of diabetes in adults in Australia. Diabetes Research and Clinical Practice, 99, 385-390. doi: 10.1016/j.diabres.2012.12.002

Lim, S. S., Norman, R. J., Clifton, P. M., \& Noakes, M. (2009). Psychological effects of prescriptive versus general lifestyle advice for weight loss in young women. Journal of the American Dietetic Association, 109, 1917-1921. doi: 10.1016/j.jada.2009.08.008

Lowe, M. R., Butryn, M. L., Didie, E. R., Annunziato, R. A., Thomas, J. G., Crerand, C. E., . . Halford, J. (2009). The Power of Food Scale: A new measures of the psychological influence of the food environment. Appetite, 53, 114-118. doi: 10.1016/j.appet.2009.05.016.

Mak, K. K., \& Lai, C. M. (2012). Assessment of dietary restraint: Psychometric properties of the Revised Restraint Scale in Hong Kong adolescents. International Journal of Behavioural Medicine, 19, 199-207. doi:10.1007/s12529-011-9161-x

Mann, T., Tomiyama, A. J., Westling, E., Lew, A. M., Samuels, B., \& Chatman, J. (2007). Medicare's search for effective obesity treatments: Diets are not the answer. American Psychologist, 62, 220-233. doi: 10.1037/0003-066X.62.3.220 
Massey, A., \& Hill, A. J. (2012). Dieting and food craving. A descriptive, quasi-prospective study. Appetite, 58, 781-785. doi: 10.1016/j.appet.2012.01.020

Moreneo, S., Rodríguez, S., Fernandez, M. C., Tamez, J., \& Cepeda-Benito, A. (2008). Clinical validation of the trait and state versions of the Food Craving Questionnaire. Assessment, 15, 375-387. doi: 10.1177/1073191107312651

National Health and Medical Research Council. (2013). Clinical practice guidelines for the management of overweight and obesity in adults, adolescents and children in Australia. Melbourne: National Health and Medical Research Council.

Nguyen, T., \& Lau, D. C. (2012). The obesity epidemic and its impact on hypertension. Canadian Journal of Cardiology, 28, 326-333. doi:10.1016/j.cjca.2012.01.001

Oschman, J. (2006). Trauma energetics. Journal of Bodywork and Movement Therapies, 10, 21.

Overduin, J., \& Jansen, A. (1996). Food cue reactivity in fasting and non-fasting subjects. European Eating Disorders Review, 4, 249-259.

Painot, D., Jotterand, S., Kammer, A., Fossati, M., \& Golay, A. (2001). Simultaneous nutritional cognitive behavioural therapy in obese adults. Patient Education and Counselling, 42, 47-52. doi:10.1016/S0738-3991(00)00092-6

Reilly, J. J., \& Kelly, J. (2011). Long-term impact of overweight and obesity in childhood and adolescence on morbidity and premature mortality in adulthood: Systematic review. International Journal of Obesity, 35, 891-898. doi:10.1038/ijo.2010.222

Rogers, P. J., \& Smit, H. J. (2000). Food craving and food 'addiction': A critical review of the evidence from a biopsychosocial perspective. Pharmacology Biochemistry and Behavior, 66, 3-14.

Rowe, J. (2005). The effects of EFT on long-term psychological symptoms. Counselling and Clinical Psychology, 2, 104-111 
Schuurmans, J., Comijs, H., Emmelkamp, P. M., Weijnen, I. J., van den Hout, M., \& van Dyck, R. (2009). Long-term effectiveness and prediction of treatment outcome in cognitive behavioral therapy and sertraline for late-life anxiety disorders. International Psychogeriatrics, 21, 1148-1159. doi: s1041610209990536

Shaw, K., O’Rourke, P., Del Mar, C., \& Kenardy, J. (2005). Psychological interventions for overweight or obesity. Cochrane Database of Systematic Review, 2, 456-471. Doi: 10.1002/14651858.CD003818.pub2

Stapleton, P., Devine, S., Chatwin, H., Porter, B., \& Sheldon, T. (2014). A feasibility study: emotional freedom techniques for depression in australian adults. Current Research in Psychology, 5, 19-33.

Stapleton, P., Sheldon, T., Porter, B. (2012). Clinical benefits of emotional freedom techniques on food cravings as 12-months follow-up: A randomized controlled trial. Energy Psychology, 4, 1-12.

Stapleton, P. B., Sheldon, T., Porter, B., \& Whitty, J. (2011). A randomized clinical trial of a meridian-based intervention for food cravings with six-month follow-up. Behaviour Change, 28, 1-16.

Steel, D., Kemps, E., \& Tiggemann, M. (2006). Effects of hunger and visuo-spatial interference on imagery-induced food cravings. Appetite, 46, 36-40. doi: 10.1016/j.appet.2005.11.001

Strategic Advantages. (2000). Symptom Assessment - 45 questionnaire (SA-45). New York: Multi-Health Systems Inc.

Stroebe, W., Papies, E.K., \& Aarts, H. (2008). From homeostatic to hedonic theories of eating: Self-regulatory failure in food-rich environments. Applied Psychology: An International Review, 57, 172-193. doi: 10.1111/j.1464-0597.2008.00360

Svetkey, L. P., Stevens, V. J., Brantley, P. J., Appel, L. J., Hollis, J. F., Loria, C. M., \& . . 
Weight Loss Maintenance Collaborative Research Group. (2008). Comparison of strategies for sustaining weight loss: The weight loss maintenance randomized controlled trial. Journal of the American Medical Association, 299, 1139-1148.

Tiggemann, M., \& Kemps, E. (2005). The phenomenology of food cravings: The role of mental imagery. Appetite, 45, 305-313.

Turk, M. W., Yang, K., Hravnak, M., Sereika, S. M., Ewing, L. J., \& Burke, L. E. (2009). Randomized clinical trials of weight loss maintenance: A review. Journal of Cardiovascular Nursing, 24, 58-80.

Wadden, T. A., Steen, S. N., Wingate, B. J., \& Foster, G. D. (1996). Psychosocial consequences of weight reduction: How much weight loss is enough? American Journal of Clinical Nutrition, 63, 461-465.

Wansink, B., Cheney, M. M., \& Chan, N. (2003). Exploring comfort food preferences across age and gender. Physiology \& Behavior, 79, 739-747. doi: 10.1016/S00319384(03)00203-8

Wardle, J., Steptoe, A., Oliver, G., \& Lipsey, Z. (2000). Stress, dietary restraint, and food intake. Journal of Psychosomatic Research, 48, 195-202. doi:10.1016/S00223999(00)00076-3

Weingarten, H. P., \& Elston, D. (1991). Food cravings in a college population. Appetite, 17, $167-175$.

Wells, S., Polglase, K., Andrews, H. B., Carrington, P. \& Baker, A. H. (2003). Evaluation of a meridian-based intervention, emotional freedom techniques (EFT), for reducing specific phobias of small animals. Journal of Clinical Psychology, 59, 943- 966.

White, M. A., \& Grilo, C. (2005). Psychometric properties of the Food Craving Inventory among obese patients and binge eating disorder. Eating Behaviours, 6, 239-245. doi:10.1016/j.eatbeh.2005.01.001 
White, M. A., Whisenhunt, B. L., Williamson, D. A., Greenway, F. L., \& Netemeyer, R. G. (2002). Development and validation of the Food-Craving Inventory. Obesity, 10, 107114. doi: 10.1038/oby.2002.17

Wilson, G. T., \& Fairburn, C. G. (2007). Eating disorders. In P. E. Nathan, \& J. M. Gordon (Eds.), Guide to treatments that work - third edition (pp. 559-592). New York: Oxford University Press.

Withrow, D., \& Alter, D. A. (2010). The economic burden of obesity worldwide: A systematic review of the direct costs of obesity. Obesity, 12, 131-141. doi: 10.1111/j.1467-789X.2009.00712.x

Witt, A. A., \& Rowe, M. R. (2014). Hedonic hunger and binge eating among women with eating disorders. International Journal of Eating Disorders, 47, 273-280. doi:10.1002/eat.22171

World Health Organisation. (2014). Obesity and overweight fact sheet. Retrieved from http://www.who.int/en

Wurtman, R. J., \& Wurtman, J. J. (1995). Brain serotonin, carbohydrate craving, obesity and depression. Obesity Research, 3, 477-480.

Zellner, D. A., Garriga-Trillo, A., Centeno, S., \& Wadsworth, E. (2004). Chocolate craving and the menstrual cycle. Appetite, 42, 119-121. doi: 10.1016/j.appet.2003.11.004 
Appendix A

Relevant Tables

Table 1

Summary of Demographic Information

\begin{tabular}{|c|c|c|c|}
\hline Variable & $\begin{array}{l}\text { EFT Group } \\
\quad(n=52)\end{array}$ & $\begin{array}{l}\text { CBT Group } \\
(n=34)\end{array}$ & $\begin{array}{c}\text { Community Sample } \\
(n=92)\end{array}$ \\
\hline \multicolumn{4}{|l|}{ Gender } \\
\hline Female & $46(88 \%)$ & \multirow{2}{*}{$\begin{array}{c}31 \text { (91\%) } \\
3(8 \%)\end{array}$} & 78 (84.8\%) \\
\hline Male & $6(11 \%)$ & & $14(15.2 \%)$ \\
\hline \multicolumn{4}{|l|}{ Age } \\
\hline$\leq 25$ years & $5(9 \%)$ & $1(2 \%)$ & $24(26.1 \%)$ \\
\hline 26 to 30 years & $5(9 \%)$ & $3(8 \%)$ & $5(5.4 \%)$ \\
\hline 31 to 40 years & 13 (25\%) & $10(29 \%)$ & $23(25.0 \%)$ \\
\hline 41 to 50 years & $7(13 \%)$ & $15(44 \%)$ & $21(22.8 \%)$ \\
\hline 51 to 60 years & $14(26 \%)$ & $5(14 \%)$ & $11(12.0 \%)$ \\
\hline$\geq 61$ years & $8(15 \%)$ & 0 & $8(8.7 \%)$ \\
\hline Mean BMI (SD) & $33.3(6.52)$ & $34.31(5.95)$ & $23.64(4.43)$ \\
\hline Overweight & $9(17 \%)$ & $8(23 \%)$ & \\
\hline Class 1 BMI $30-34.99$ & $15(28 \%)$ & $10(29 \%)$ & \\
\hline Class II BMI 35 - 39.99 & $15(28 \%)$ & $7(20 \%)$ & \\
\hline Class III BMI 40+ & $5(9 \%)$ & $823 \%)$ & \\
\hline Married & $25(48 \%)$ & $15(46 \%)$ & 37 (40.2\%) \\
\hline Education post secondary school & 27 (51\%) & $13(40 \%)$ & $82(90 \%)$ \\
\hline Employed & 31 (59\%) & 25 (78\%) & $60(65 \%)$ \\
\hline \multicolumn{4}{|l|}{ Income (AUD\$) } \\
\hline$<\$ 10,000$ & $12(23 \%)$ & $7(20 \%)$ & $10(10 \%)$ \\
\hline$\$ 10,001$ to $\$ 40,000$ & $15(28 \%)$ & $14(41 \%)$ & $16(17 \%)$ \\
\hline$\$ 40,001$ to $\$ 60,000$ & $7(13 \%)$ & $5(14 \%)$ & $21(22.8 \%)$ \\
\hline$\$ 60,001$ to $\$ 80,000$ & $3(5 \%)$ & $3(8 \%)$ & 10 10\%) \\
\hline$\$ 80,001$ to $\$ 100,000$ & $5(9 \%)$ & 0 & $15(16 \%)$ \\
\hline$>\$ 100,000$ & $10(19 \%)$ & $5(14 \%)$ & 32 (35\%) \\
\hline
\end{tabular}


Table 2

Group Means and Standard Deviations for the Dependent Variables across Time, including 95\% Confidence Intervals for the Collapsed Group Mean Scores.

\begin{tabular}{|c|c|c|c|c|c|c|c|c|}
\hline \multirow[b]{2}{*}{ Variable } & \multicolumn{2}{|c|}{ EFT Group } & \multicolumn{2}{|c|}{ CBT Group } & \multicolumn{2}{|c|}{ Collapsed Total } & \multicolumn{2}{|c|}{$95 \% \mathrm{CI}$} \\
\hline & $M$ & $S D$ & $M$ & $S D$ & $M$ & $S D$ & Lower & Upper \\
\hline FCI (Baseline) & 61.09 & 19.90 & 68.54 & 16.61 & 64.29 & 18.73 & 59.26 & 69.31 \\
\hline POF (Baseline) & 69.84 & 21.84 & 78.00 & 17.00 & 73.34 & 20.16 & 67.97 & 78.74 \\
\hline RRS (Baseline) & 33.22 & 6.91 & 33.96 & 5.59 & 33.54 & 6.54 & 31.84 & 35.23 \\
\hline BMI (Baseline) & 33.37 & 6.95 & 34.81 & 6.57 & 33.99 & 6.77 & 32.18 & 35.80 \\
\hline FCI (Post) & 47.97 & 12.89 & 49.75 & 19.59 & 48.73 & 15.97 & 44.46 & 53.01 \\
\hline POF (Post) & 53.63 & 21.84 & 58.25 & 18.01 & 55.61 & 19.96 & 50.26 & 60.95 \\
\hline RRS (Post) & 31.31 & 6.14 & 29.29 & 6.93 & 30.45 & 6.52 & 28.70 & 32.19 \\
\hline BMI (Post) & 32.87 & 7.03 & 34.14 & 6.85 & 33.41 & 6.92 & 31.56 & 35.27 \\
\hline FCI (6-mth follow-up) & 47.41 & 12.32 & 52.25 & 18.65 & 49.48 & 15.07 & 45.45 & 53.52 \\
\hline POF (6-mth follow-up) & 50.19 & 27.03 & 56.46 & 19.77 & 52.88 & 24.19 & 46.40 & 59.35 \\
\hline RRS (6-mth follow-up) & 28.88 & 9.36 & 29.92 & 6.78 & 29.32 & 8.30 & 27.10 & 31.54 \\
\hline BMI (6-mth follow-up) & 33.40 & 14.10 & 33.59 & 6.41 & 33.48 & 11.37 & 30.44 & 36.53 \\
\hline FCI (12-mth follow-up) & 50.34 & 14.43 & 52.42 & 18.65 & 51.23 & 16.24 & 46.88 & 55.58 \\
\hline POF (12-mth follow-up) & 51.84 & 23.36 & 53.17 & 21.74 & 52.41 & 22.49 & 46.36 & 58.43 \\
\hline RRS (12-mth follow-up) & 25.00 & 11.78 & 27.87 & 7.98 & 26.23 & 10.34 & 23.46 & 29.00 \\
\hline BMI (12-mth follow-up) & 33.71 & 14.44 & 33.35 & 6.54 & 33.55 & 11.64 & 33.55 & 30.44 \\
\hline
\end{tabular}

Note. FCI $=$ Food Craving Inventory. POF $=$ Power of Food Scale. RRS = Revised Restraint Scale. BMI = Body Mass Index. 95\% CI = 95\% confidence intervals for the total mean scores (i.e., collapsed across CBT and EFT). 
Table 3

Simple Effects Analyses for Time Using Collapsed Group Mean Scores.

\begin{tabular}{|c|c|c|c|}
\hline Variable + Time Point & $p$ & Cohen's $d$ & Effect Size Description \\
\hline Food Cravings & $<.001$ & & \\
\hline $1 \rightarrow 2$ & $<.001$ & .89 & Large \\
\hline $1 \rightarrow 3$ & $<.001$ & .87 & Large \\
\hline $1 \rightarrow 4$ & $<.001$ & .74 & Medium-Large \\
\hline $2 \rightarrow 3$ & .682 & - & - \\
\hline $3 \rightarrow 4$ & .311 & - & - \\
\hline Power of Food & $<.001$ & & \\
\hline $1 \rightarrow 2$ & $<.001$ & .83 & Large \\
\hline $1 \rightarrow 3$ & $<.001$ & .92 & Large \\
\hline $1 \rightarrow 4$ & $<.001$ & .98 & Large \\
\hline $2 \rightarrow 3$ & .367 & - & - \\
\hline $3 \rightarrow 4$ & .581 & - & - \\
\hline Restraint & .028 & & \\
\hline $1 \rightarrow 2$ & $<.001$ & .48 & Medium \\
\hline $1 \rightarrow 3$ & $<.001$ & .57 & Medium \\
\hline $1 \rightarrow 4$ & $<.001$ & .85 & Large \\
\hline $2 \rightarrow 3$ & .407 & - & \\
\hline $3 \rightarrow 4$ & .005 & .33 & Small-Medium \\
\hline BMI & .722 & & \\
\hline $1 \rightarrow 2$ & .061 & - & - \\
\hline $1 \rightarrow 3$ & .657 & - & - \\
\hline $1 \rightarrow 4$ & .674 & - & - \\
\hline $2 \rightarrow 3$ & .996 & - & - \\
\hline $3 \rightarrow 4$ & .869 & - & - \\
\hline
\end{tabular}

Note. 1 = pre-intervention, 2 = post-intervention, 3 = 6-mth follow-up, 4 = 12-mth follow-up. $p=$ significance value (to 3 decimal points). 
Table 4

Means and Standard Deviations for the Intervention Groups Compared to the Non-Clinical

Sample, including Univariate Between-Subjects Analyses.

\begin{tabular}{|c|c|c|c|c|c|c|c|c|}
\hline \multirow[b]{2}{*}{ Variable } & \multicolumn{2}{|c|}{ EFT Group } & \multicolumn{2}{|c|}{ CBT Group } & \multicolumn{2}{|c|}{ Community Sample } & \multicolumn{2}{|c|}{ Between-group effects } \\
\hline & $M$ & $S D$ & $M$ & $S D$ & $M$ & $S D$ & $F$ & $p$ \\
\hline FCI (Baseline) & 61.09 & 19.90 & 68.54 & 16.61 & 49.03 & 14.81 & 16.65 & $<.001$ \\
\hline POF (Baseline) & 69.84 & 21.84 & 78.00 & 17.00 & 46.05 & 16.32 & 42.59 & $<.001$ \\
\hline RRS (Baseline) & 33.22 & 6.91 & 33.96 & 5.59 & 24.37 & 6.36 & 36.17 & $<.001$ \\
\hline BMI (Baseline) & 33.37 & 6.95 & 34.81 & 6.57 & 23.64 & 4.43 & 63.68 & $<.001$ \\
\hline FCI (Post) & 47.97 & 12.89 & 49.75 & 19.59 & - & - & .10 & .905 \\
\hline POF (Post) & 53.63 & 21.84 & 58.25 & 18.01 & - & - & 5.49 & .005 \\
\hline RRS (Post) & 31.31 & 6.14 & 29.29 & 6.93 & - & - & 16.32 & $<.001$ \\
\hline BMI (Post) & 32.87 & 7.03 & 34.14 & 6.85 & - & - & 55.19 & $<.001$ \\
\hline FCI (6-mth follow-up) & 47.41 & 12.32 & 52.25 & 18.65 & - & - & .74 & .478 \\
\hline POF (6-mth follow-up) & 50.19 & 27.03 & 56.46 & 19.77 & - & - & 2.80 & .064 \\
\hline RRS (6-mth follow-up) & 28.88 & 9.36 & 29.92 & 6.78 & - & - & 8.45 & $<001$ \\
\hline BMI (6-mth follow-up) & 33.40 & 14.10 & 33.59 & 6.41 & - & - & 27.51 & $<.001$ \\
\hline FCI (12-mth follow-up) & 50.34 & 14.43 & 52.42 & 18.65 & - & - & .48 & .620 \\
\hline POF (12-mth follow-up) & 51.84 & 23.36 & 53.17 & 21.74 & - & - & 1.99 & .140 \\
\hline RRS (12-mth follow-up) & 25.00 & 11.78 & 27.87 & 7.98 & - & - & 1.80 & .169 \\
\hline BMI (12-mth follow-up) & 33.71 & 14.44 & 33.35 & 6.54 & - & - & 26.88 & $<.001$ \\
\hline
\end{tabular}

Note. FCI = Food Craving Inventory. POF = Power of Food Scale. RRS = Revised Restraint Scale. BMI = Body Mass Index. 
Appendix B

Figures - Consort Diagram

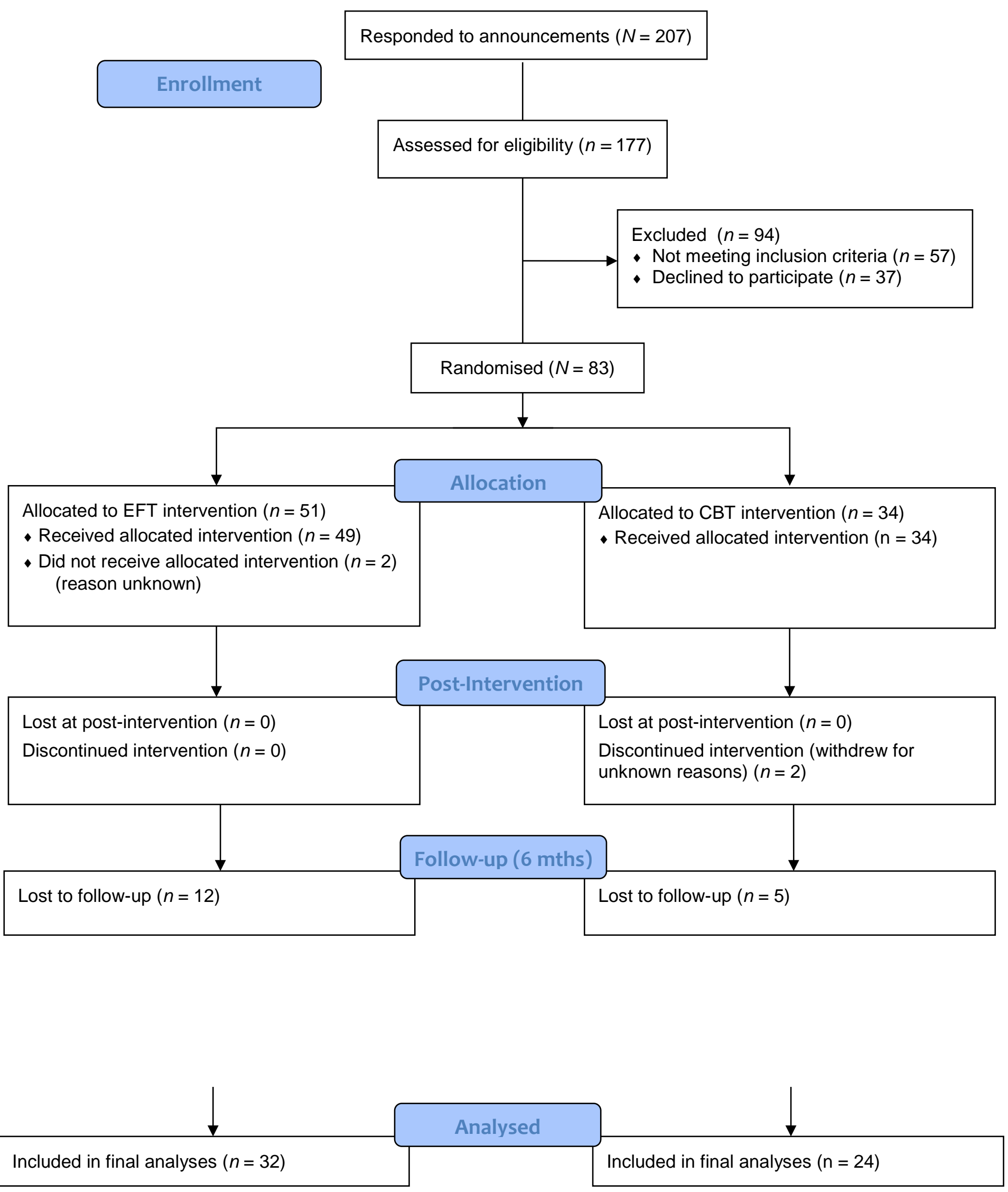




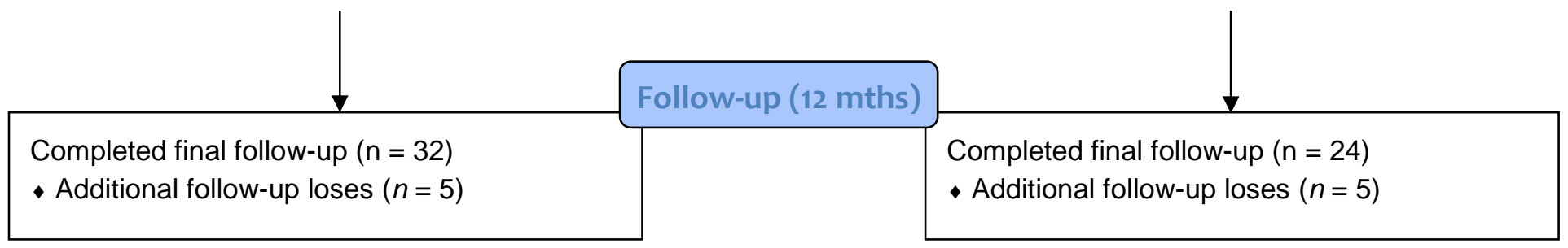

